\title{
First Results from ARTEMIS, a New Two-Spacecraft Lunar Mission: Counter-Streaming Plasma Populations in the Lunar Wake
}

\author{
J.S. Halekas • V. Angelopoulos • D.G. Sibeck · K.K. Khurana · C.T. Russell • \\ G.T. Delory · W.M. Farrell · J.P. McFadden · J.W. Bonnell · D. Larson · R.E. Ergun • \\ F. Plaschke $\cdot$ K.H. Glassmeier
}

Received: 14 July 2010 / Accepted: 14 December 2010

(C) The Author(s) 2011. This article is published with open access at Springerlink.com

\begin{abstract}
We present observations from the first passage through the lunar plasma wake by one of two spacecraft comprising ARTEMIS (Acceleration, Reconnection, Turbulence, and Electrodynamics of the Moon's Interaction with the Sun), a new lunar mission that re-tasks two of five probes from the THEMIS magnetospheric mission. On Feb 13, 2010, ARTEMIS probe P1 passed through the wake at $\sim 3.5$ lunar radii downstream from the Moon, in a region between those explored by Wind and the Lunar Prospector, Kaguya, Chandrayaan, and Chang'E missions. ARTEMIS observed interpenetrating proton, alpha particle, and electron populations refilling the wake along magnetic field lines from both flanks. The characteristics of these distributions match expectations from self-similar models of plasma expansion into vacuum, with an asymmetric character likely driven by a combination of a tilted interplanetary magnetic field and an anisotropic incident solar wind electron population. On this flyby, ARTEMIS provided unprecedented measurements of the interpenetrating beams of both electrons and ions naturally produced by the filtration and acceleration effects of electric fields set up during the refilling process. ARTEMIS also measured electrostatic oscillations closely correlated with counter-streaming electron beams in the wake, as previ-
\end{abstract}

J.S. Halekas (凶) · G.T. Delory · J.P. McFadden · J.W. Bonnell · D. Larson Space Sciences Laboratory, University of California, Berkeley, CA 94720, USA e-mail: jazzman@ssl.berkeley.edu

J.S. Halekas · G.T. Delory · W.M. Farrell

NASA's Lunar Science Institute, NASA Ames Research Center, Moffett Field, CA 94035, USA

V. Angelopoulos · K.K. Khurana · C.T. Russell

Institute of Geophysics and Planetary Physics, UCLA, Los Angeles, CA 90095, USA

D.G. Sibeck · W.M. Farrell

NASA Goddard Space Flight Center, Greenbelt, MD 20771, USA

R.E. Ergun

Laboratory for Atmospheric and Space Physics, University of Colorado, Boulder, CO 80303, USA

F. Plaschke · K.H. Glassmeier

Institut für Geophysik und Extraterrestrische Physik, Braunschweig, Germany

Published online: 20 January 2011 
ously hypothesized but never before directly measured. These observations demonstrate the capability of the comprehensively instrumented ARTEMIS spacecraft and the potential for new lunar science from this unique two spacecraft constellation.

Keywords Moon $\cdot$ Lunar wake $\cdot$ Counter-streaming distributions

\section{Introduction}

In contrast to the rather static lunar interior, the Moon's plasma environment is highly dynamic, with the surface fully exposed to the ever-changing solar wind and magnetospheric charged particles. These plasma inputs, which vary by orders of magnitude in velocity, density, and temperature, especially during active solar periods, drive a commensurately variable Moon-plasma interface, which we have studied since the Apollo era (Ness 1972; Schubert and Lichtenstein 1974). Charged particle and photon impacts generate much of the tenuous lunar exosphere and play a role in its loss. They also drive highly variable surface charging on both the sunlit and shadowed hemispheres of the Moon (Freeman et al. 1973; Halekas et al. 2008b). Relatively small and weak, but widely distributed, lunar crustal magnetic anomalies (Dyal et al. 1974) produce perhaps some of the smallest collisionless shocks and magnetospheres in the solar system (Russell and Lichtenstein 1975; Lin et al. 1998; Halekas et al. 2008a; Wieser et al. 2010; Saito et al. 2010). These and other elements of the plasma environment couple to the lunar surface and exosphere, and very likely to lunar dust, in ways not yet completely understood.

The lunar plasma wake exhibits a host of particularly fascinating physical processes. Solar wind ions impact the dayside lunar surface, implanting or scattering, and leaving a void downstream from the Moon. The resulting plasma void has vanishingly low plasma density, and refills only slowly as solar wind ions flow at supersonic speeds past the Moon. The pressure gradient across the wake boundary creates diamagnetic current systems that reduce the magnetic field magnitude in the expansion region near the wake boundary and enhance the field in the central wake (Colburn et al. 1967; Ness et al. 1967; Owen et al. 1996; Halekas et al. 2005). Charged particles refill the wake along magnetic field lines, with the higher thermal speed of the electrons driving a charge-separation electric field that approximately maintains quasi-neutrality while slowing electron expansion and accelerating ions into the wake. Theoretical studies and simulations of this general plasma process have shown that this leads to a rarefaction wave propagating back into the plasma, particle expansion into the vacuum with a velocity related to the ion sound speed, and an exponentially decreasing density on the vacuum side of the interface (Crow et al. 1975; Denavit 1979; Samir et al. 1983). Observations from Wind first demonstrated that this process operates to refill the lunar wake (Ogilvie et al. 1996; Clack et al. 2004), and numerous simulations have since refined our understanding (Farrell et al. 1998; Birch and Chapman 2001a, 2001b; Kallio 2005; Trávnicek and Hellinger 2005; Kimura and Nakagawa 2008). More recently, we have learned that ions can also refill the wake perpendicular to the magnetic field direction, with gyrating protons (Type-I entry) and re-picked up protons scattered from the dayside surface (Type-II entry) both capable of penetrating deep into the wake cavity (Nishino et al. 2009a, 2009b; Holmström et al. 2010). We do not yet fully understand the relative importance of parallel and perpendicular refilling processes in the wake, or how this varies as a function of distance downstream from the Moon.

Many open questions remain about the lunar plasma environment, and each mission to the Moon to date has made new discoveries that have changed our understanding of 
the Moon-plasma interaction. The latest generation of international lunar missions, namely Kaguya, Chandrayaan, and Chang'E, observed many new phenomena, including magnetic shielding of surface regions (Saito et al. 2010; Wieser et al. 2010), solar wind scattering from the dayside surface (Saito et al. 2008; Wieser et al. 2009), gyrating and re-picked up scattered protons refilling the wake (Nishino et al. 2009a, 2009b), and scattered protons interacting with the wake electric field (Nishino et al. 2009a; Wang et al. 2010). Even now, many components of the lunar plasma environment remain either incompletely understood or unobserved.

Given the opportunity to add to our understanding of this fascinating environment, we retasked two of the probes from the THEMIS (Time History of Events and Macroscale Interactions During Substorms) five-spacecraft magnetospheric constellation mission (Angelopoulos 2008) on an extended mission to the Moon, as a new lunar mission named ARTEMIS (Acceleration, Reconnection, Turbulence, and Electrodynamics of the Moon's Interaction with the Sun). These comprehensively instrumented spacecraft, currently in transition orbits, will eventually enter into elliptical orbits around the Moon, providing unprecedented two-point measurements over a range of altitudes from $\sim 100-10,000 \mathrm{~km}$, with a variety of orbital phasings and probe separations. In this paper, we describe observations from the first lunar flyby through the lunar wake by ARTEMIS P1 (formerly THEMIS-B).

\section{Overview of the Feb 13, 2010 ARTEMIS Flyby}

The first flyby of the lunar wake by ARTEMIS P1 took place on February 13, 2010. We show observations from several of the ARTEMIS instruments in Fig. 1, and an overview of the geometry of the flyby in Fig. 2. The spacecraft passed through the plasma wake $\sim 3.5$ lunar radii $\left(R_{M}=1738 \mathrm{~km}\right)$ downstream from the Moon, in a region between those observed by Wind and the more recent low-altitude Lunar Prospector, Kaguya, Chang'E, and Chandrayaan missions. The GSE position of the Moon was roughly [62.2, -8.7, 2.5] earth radii, well upstream from the terrestrial magnetosphere and certainly not connected to the terrestrial foreshock, given the large $z$-component of the magnetic field. The ARTEMIS P1 probe traveled through the wake at an oblique angle, with the orbit trajectory fortuitously aligned nearly along the prevailing magnetic field direction. This afforded us an excellent opportunity to measure parallel wake expansion processes along magnetic field lines. The combination of unfavorable non-perpendicular magnetic field geometry and distance from the Moon apparently precluded us from seeing any effects of Type-I proton gyration entry or Type-II pickup entry perpendicular to field lines (Nishino et al. 2009a, 2009b).

Ion observations by the ESA instrument (McFadden et al. 2008) indicate bi-directional penetration of ions into the wake along magnetic field lines, with roughly exponentially decreasing density in the wake, bounded by an external rarefaction wave, as predicted by models of plasma expansion into vacuum (Samir et al. 1983) and previously observed by Wind (Ogilvie et al. 1996). The characteristic energy loss/gain of the ions on the entry/exit sides of the wake results from ion acceleration along tilted magnetic field lines, which subtracts/adds to the solar wind velocity on the entry/exit sides, as clearly seen in the velocity components. We discuss ion observations further in Sect. 4.

During this first wake flyby, the ESA instrument operated in magnetospheric mode (McFadden et al. 2008), allowing full energy and angular coverage of ions, but with reduced resolution $\left(22.5^{\circ}\right.$ angular resolution, $32 \%$ fractional energy resolution). In this mode, the instrument can underestimate the magnitude of the solar wind ion density due to the slower energy sweep, and the transverse components of the ion velocity can have small errors because of the reduced angular resolution. Nonetheless, we have compared integrated ion and 


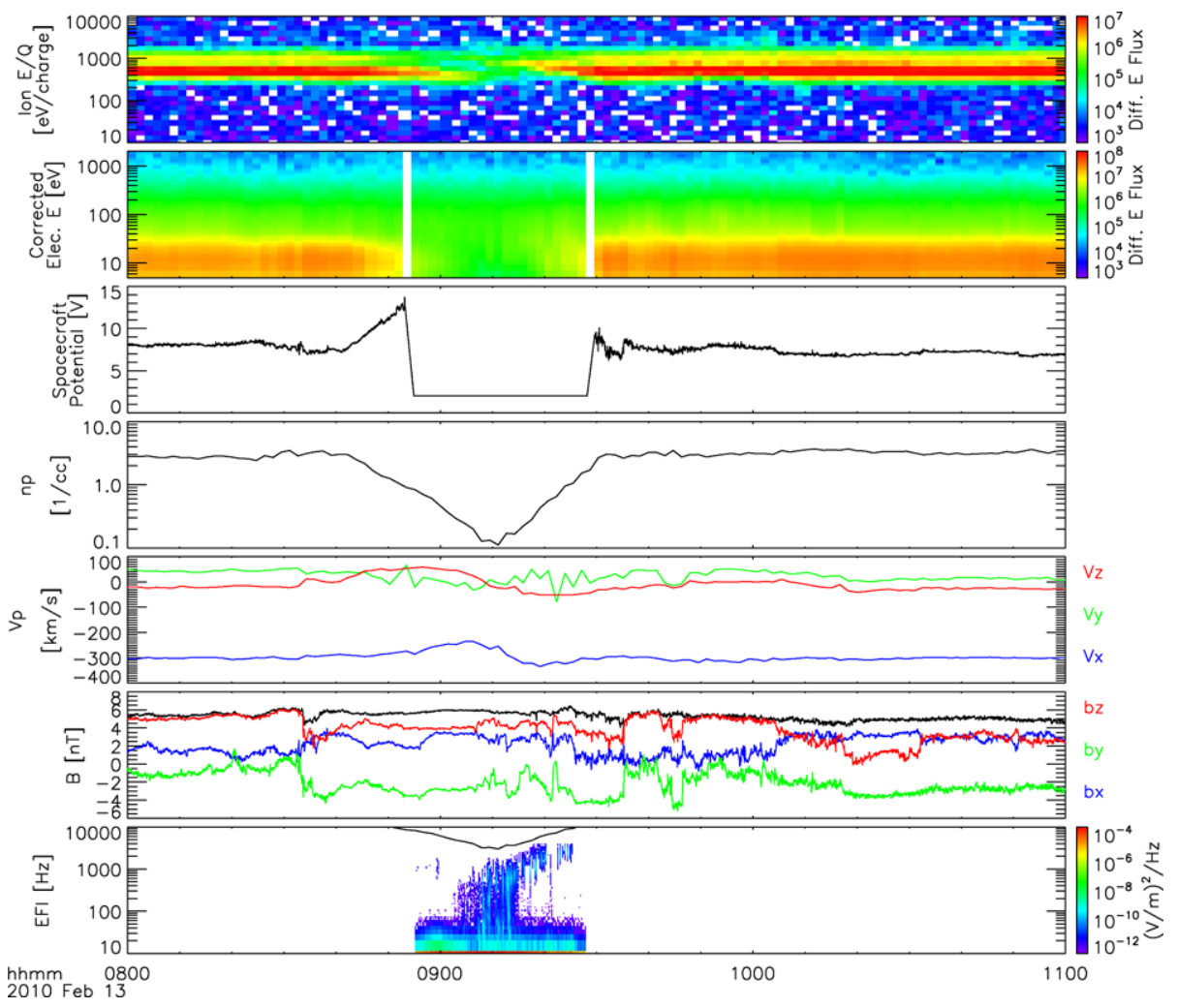

Fig. 1 ARTEMIS observations of the lunar plasma wake on Feb 13, 2010. Panels show omni-directional ion and electron energy-time spectrograms $\left[\mathrm{eV} /\left(\mathrm{cm}^{2} \mathrm{~s} \mathrm{sreV}\right)\right]$, spacecraft potential (measured in sunlight, inferred from ion/electron comparisons in shadow), ion density and velocity moments in Selenocentric Solar Ecliptic (SSE) coordinates, magnetic field in SSE coordinates, and an electric field wave power frequency-time spectrogram (black curve shows electron plasma frequency). Electron spectra are corrected for the effects of spacecraft charging. Data gaps (white strips) indicate times when the spacecraft potential changed too rapidly to apply corrections

electron density and velocity moments outside the wake and found good agreement, indicating accurate measurements of bulk plasma parameters. Future flybys with the ESA instruments in solar wind mode will provide higher resolution measurements of the distributions of solar wind ions as they refill the wake.

In sunlight, the EFI instrument (Bonnell et al. 2008) measures the spacecraft potential (see Fig. 1), allowing us to correct for the effects of spacecraft charging. Just inside the plasma wake (as indicated by the reduction in plasma density, indicating the extent of the rarefaction wave), but outside of the Moon's penumbra, EFI observes an increase in spacecraft potential resulting from the reduction in plasma density. Inside the Moon's penumbra, we tested a new biasing scheme for the EFI probes, with the aim of controlling the spacecraft potential. This biasing scheme successfully moderated spacecraft charging, resulting in a spacecraft potential of approximately two volts positive, as derived from comparison of ion and electron density and velocity moments. This small and well-estimated spacecraft potential allows us to correct for spacecraft charging even in the shadowed wake region, providing a critically important capability for quantitatively analyzing charged particle observations. 
Fig. 2 Geometry of the Feb 13, 2010 ARTEMIS wake flyby, in aberrated SSE coordinates, assuming a perfectly cylindrical lunar wake extended in the direction of the aberrated solar wind flow. We color code the spacecraft track by the log of the ion density, and show magnetic field vectors at the times indicated
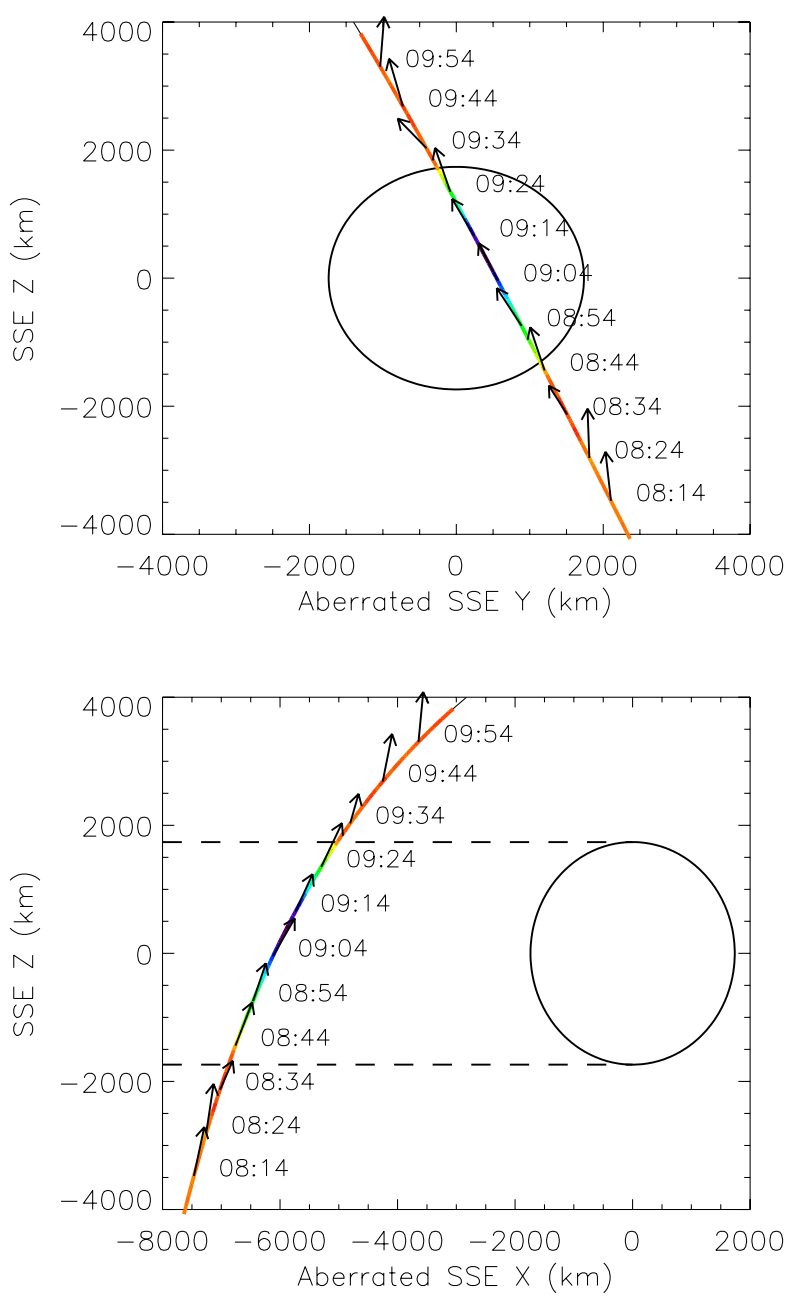

To demonstrate this capability, we display electron spectra corrected for the effects of spacecraft charging in Fig. 1, showing a smoothly varying spectrum as a function of time, with no artificial discontinuities at the light/shadow boundaries. Between the penumbra and the umbra, the spacecraft potential changed rapidly, and we did not attempt to correct these electron spectra (blank regions in electron spectrogram in Fig. 1). Electrons, like ions, drop out as the spacecraft travels deeper into the wake, leaving only a residual halo/strahl population in the central wake. We discuss electron observations further in Sect. 5.

EFI also measures the electric field component of plasma waves. During this flyby, as shown in the bottom panel of Fig. 1, EFI observed electric field oscillations extending almost up to the electron plasma frequency, primarily on the exit side of the wake. During this same time period, we observed no magnetic field fluctuations with the SCM instrument (Roux et al. 2008), identifying these oscillations as primarily electrostatic. We discuss possible sources for these waves further in Sect. 5.

The FGM instrument (Auster et al. 2008) measured the vector magnetic field. In shadow, the spacecraft loses the sun pulse, and its spin period drifts slightly due to thermal effects. 
Fig. 3 Magnetic field vector data time series measured by Wind (time-shifted according to the solar wind velocity and spatial separation of the two spacecraft) and ARTEMIS, in GSE coordinates, and a comparison of magnetic field magnitudes measured by the two spacecraft. Dashed lines show the extent of the plasma wake as indicated by ion density

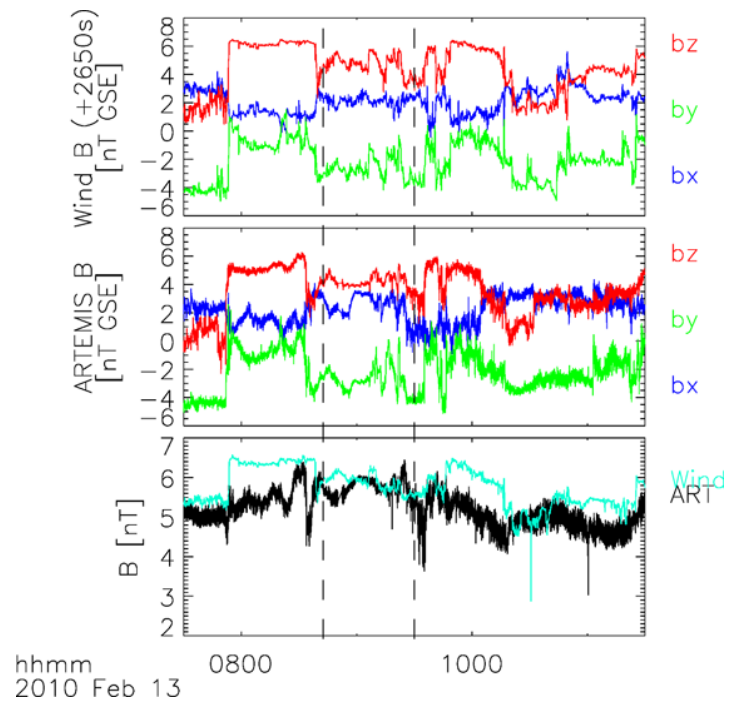

The FGM team has utilized magnetic field data, along with modeling of the thermal contraction of the spacecraft, to derive the actual spacecraft spin period in shadow, allowing us to correctly de-spin both magnetic field and plasma data. Magnetic field data thus corrected indicate a highly variable field orientation, but with a prevailing alignment between the magnetic field and the spacecraft trajectory, as shown in Fig. 2. Though we do observe several large rotations in the field, we cannot convincingly associate any of these with the wake boundary. We discuss magnetic field observations in more detail in Sect. 2.

We use the solar wind $V_{X}$ component measured by ESA, along with the $\sim 30 \mathrm{~km} / \mathrm{s}$ velocity of the Earth around the Sun, to derive an approximate aberration angle for the solar wind. The $V_{Y}$ component measured by ESA roughly matches the expected aberration; however, ESA also measures a small $V_{Z}$ component that we have not utilized in the aberration calculation. As noted above, transverse velocity components measured by ESA can have small errors in magnetospheric mode due to the low angular resolution of the binning scheme. We found that including only the $V_{Y}$ component provided a better match to the observations, with the ion density dropout that indicates the extent of the plasma wake matching the resulting assumed wake geometry.

\section{Magnetic Field Observations}

ARTEMIS FGM data indicate a highly variable magnetic field during this time period. In particular, we observe large rotations in the field just before and after the wake transit. One would like to associate these with the wake itself, but we would first have to rule out upstream influences to make such a claim. In Fig. 3, we compare Wind and ARTEMIS magnetic field data. We use the radial separation of the two spacecraft of 133 earth radii and the radial solar wind velocity measured at Wind of $320 \mathrm{~km} / \mathrm{s}$ to derive an approximate time shift of $2650 \mathrm{~s}$, which we apply to the Wind data. With this time shift, we find that Wind observes essentially all of the magnetic field signatures that ARTEMIS does, with very similar timing. Thus, all of the field rotations have a source far upstream from the Moon.

A comparison of the magnitude of the field between the two spacecraft does yield some interesting differences. For instance, ARTEMIS observes much more significant reductions 
Fig. 4 Ion spectrogram $\left[\mathrm{eV} /\left(\mathrm{cm}^{2} \mathrm{~s}\right.\right.$ sr eV) $]$ (high resolution burst data), and a series of two-component fits to the reduced ion distribution function. "A/P" indicates time periods with distributions best fit to an alpha particle plus a proton component, " $2 \mathrm{xP}$ " indicates a time period with distributions best fit to two proton components, and "No Fits" indicates a time period with no successful two-component fits.

Red, orange, blue, and black curves on both panels show modeled proton and alpha particle energies and velocities, as labeled in the figure

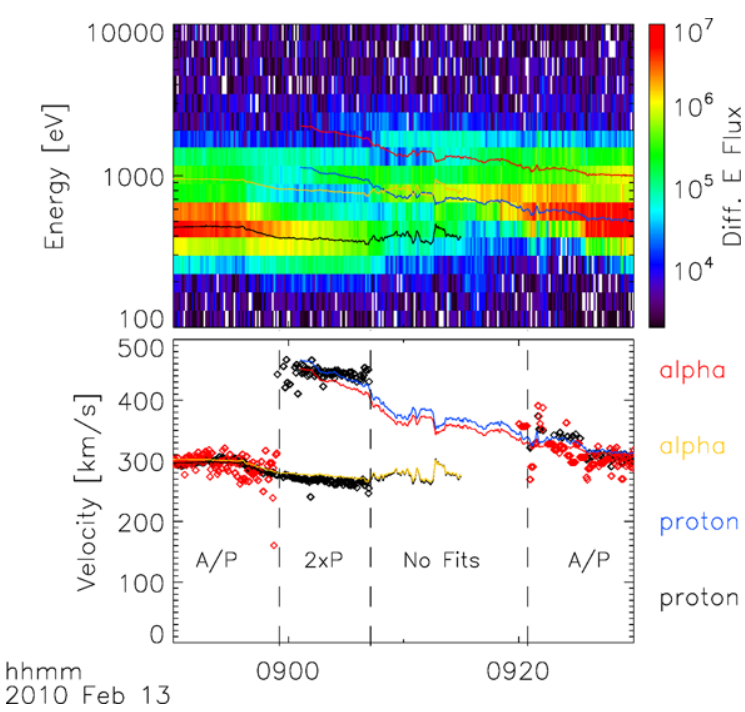

in field magnitude associated with the large rotations just before and after the wake passage. These magnetic field depressions could represent the expected diamagnetic dips in field magnitude in the expansion region of the wake. However, given their co-location with rotations also observed upstream, we do not favor such an interpretation. To make such a claim, one would have to clearly separate temporal from spatial variations, requiring more than one set of observations, preferably with both observations near the Moon. Furthermore, we find no significant increase in magnetic field magnitude in the central wake, an expected result of the diamagnetic current systems. Therefore, we cannot convincingly associate any magnetic field features seen during this wake passage with a lunar origin. In the coming years, many orbits from two identically instrumented ARTEMIS probes will allow us to sort out features associated with the Moon-plasma interaction from those generated upstream, and separate spatial from temporal variations.

\section{Ion Observations and Model Comparisons}

As discussed briefly in Sect. 2, ARTEMIS ESA data indicate ions accelerated along magnetic field lines into the wake from both sides. In Fig. 4, we show high-resolution ESA burst data for ions during the wake passage. We also display velocity magnitudes derived from two-component fits (using a gradient-expansion method with a chi square fit metric) to the reduced ion distribution function. This fitting procedure allows a separation of ion species and velocity components difficult to achieve self-consistently in a moment calculation with an instrument like ESA that does not have mass separation. Outside of the wake in the undisturbed solar wind, these fits indicate that proton and alpha particle velocities track very well, as expected. Inside the wake, though, the spectrograms and fits in Fig. 4 indicate the presence of proton and alpha particle components from both sides of the wake, with a complicated spectral structure that we can only fit to a two-component spectrum in a few regions. Wind and other spacecraft have observed accelerated protons like these refilling the wake (Ogilvie et al. 1996), and interpreted them as the result of charge-separation electric fields produced during the expansion process (Samir et al. 1983), but no one to our knowledge has previously observed alpha particle expansion into the lunar wake. 
Absent any other information, we would have a difficult time separating the four components of the ion distribution function, composed of interpenetrating protons and alphas from both sides of the wake. However, a model helps us identify the various ion components. Assuming a quasi-neutral 1-D expansion of isothermal plasma into a vacuum, one can derive so-called self-similar expansion relations (Denavit 1979; Samir et al. 1983), which depend only on the combination $s / t$, where $s$ represents the distance along the magnetic field line into the vacuum, and $t$ the expansion time (or, equivalently, the solar wind convection time past the lunar limb). In all equations, $C_{s}$ represents the ion sound speed.

$$
\begin{aligned}
& N=N_{0} \exp \left(-\left(s /\left(C_{s} t\right)+1\right)\right) \\
& \phi=-\left(T_{e} / e\right)\left(s /\left(C_{s} t\right)+1\right) \\
& V_{i}=s / t+C_{s}
\end{aligned}
$$

One can extend these relations to take into account non-Maxwellian electron distributions, with the effect of slightly increasing the potential and velocity in the wake. Lunar Prospector observed electron distributions inside and outside the wake at low altitudes, and derived electron properties and wake potentials consistent with this expanded theory (Halekas et al. 2005). Recent observations from Chandrayaan also show that this model may need some modifications near the Moon to take into account surface absorption of plasma (Futaana et al. 2010). For this case, though, we opt to use the most basic self-similar model, demonstrating how well even very simple theory matches ARTEMIS observations.

To develop our model prediction, we use spacecraft position and magnetic field data in aberrated SSE coordinates (as shown in Fig. 2) in order to calculate the convection time $t$ of the point where the magnetic field line intersects the wake boundary, and the distance $s$ along the field line into the wake from that point. Our model depends on the instantaneous magnetic field geometry, and thereby captures the asymmetry of the wake due to the tilted magnetic field. Though one-dimensional in nature, the model implicitly captures twodimensional features, since convection time maps to distance downstream from the Moon. Our model, utilizing the measured solar wind speed of $310 \mathrm{~km} / \mathrm{s}$ and an ion sound speed $C_{s}$ of $25 \mathrm{~km} / \mathrm{s}$ derived from the measured electron temperature $T_{e}$ of $\sim 6.5 \mathrm{eV}$, has no adjustable parameters. Finally, we employ an extension of the self-similar model for alpha particle behavior derived by Singh and Schunk (1982) for polar wind, which assumes that the small fraction of alpha particles do not affect the potential, but rather merely respond to it. Note that this implies that alpha particles sense a different potential than protons, since the two species travel at different velocities into the wake through an explicitly time dependent potential, and therefore we can think of the wake as filtering particles of different species.

We add the model expansion velocity determined for both protons and alpha particles to the initial solar wind velocity in vector fashion to derive the modeled total energies and velocities, which we plot over the data in Fig. 4. Our simple model matches the measured ion energy spectra and two-component velocity fits rather well, correctly reproducing the asymmetry of the wake, and the decrease/increase in energy and velocity on the entry/exit sides of the wake. The model even accurately reproduces numerous small temporal fluctuations (departures from a temporally smoothly varying spectrum) in the ion spectra that result from changes in the magnetic field geometry. This indicates that the infilling ions closely follow the instantaneous magnetic field orientation, as expected given the solar wind convection time of only 15-20 seconds from the Moon to the spacecraft. With the aid of our model, we can now clearly separate different components of the ion spectrum. We find that protons and alphas from both sides penetrate nearly all the way through the wake, with only the alpha component from the entry side proving difficult to identify in the ion spectrogram. In the 


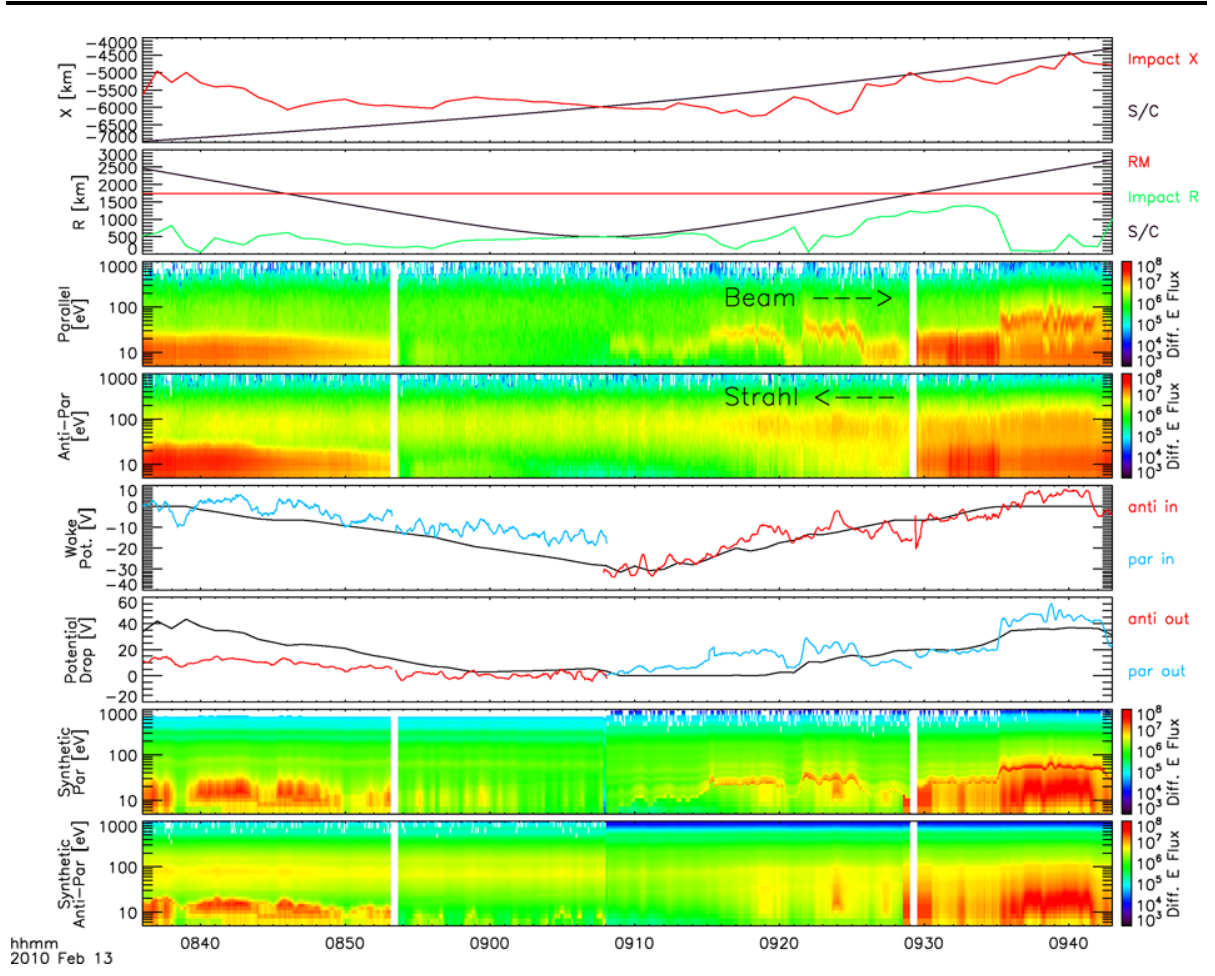

Fig. 5 The top two panels show the cylindrical coordinates of the spacecraft and the closest point to the center of the wake reached by the magnetic field line passing through the spacecraft (using a straight-line approximation). The third and fourth panels show corrected spectrograms $\left[\mathrm{eV} /\left(\mathrm{cm}^{2} \mathrm{~s} \mathrm{sreV}\right)\right]$ for parallel $\left(0-15^{\circ}\right.$ pitch angle) and anti-parallel $\left(165-180^{\circ}\right)$ traveling electrons (high resolution burst data). The fifth and sixth panels show the wake potential relative to the solar wind, and the potential drop from the deepest point into the wake along the magnetic field line to the spacecraft, as inferred from shifts in electron distribution functions. Black curves on both panels indicate model potentials. The bottom two panels show synthetic spectrograms created using the inferred potentials and four end-point electron spectra, as described in the text

central wake, at least three, and probably four, populations of ions coexist simultaneously, each with slightly different vector velocities as a result of the different electric fields felt by the various populations. This promises a rich plasma environment, with a strong likelihood of wave-particle interactions, possibly including beam-beam instabilities as postulated by Farrell et al. (1998). In the coming years, ARTEMIS's full plasma instrumentation and two-probe measurement capability will enable statistical study and clear identification of the effects of interpenetrating ions on the wake environment.

\section{Electron Observations and Model Comparisons}

As described in Sect. 2, ARTEMIS ESA data show that the bulk of the electron population drops out in the central wake, leaving a residual population sufficient only to balance the small density of interpenetrating refilling ions. The electrons that do penetrate the wake, especially the most field-aligned populations thereof, have fascinating properties. The third and fourth panels of Fig. 5 show two electron spectrograms calculated for parallel and antiparallel components of the electron population, utilizing high resolution burst data, with both 
corrected for spacecraft charging as described in Sect. 2. The potential drop set up by the wake refilling process (described in Sects. 3 and 4) excludes most of the lower-energy core electron populations from both entry and exit sides of the wake, though a residual portion of the core population penetrates to the central wake from the entry side. Meanwhile, a strong strahl component travels anti-parallel along the field lines, penetrating all the way through the wake from the exit side and traveling out the entry side. Finally, we find a beam of electrons that travels out from the center of the wake parallel to the field line, and out of the exit side of the wake. ARTEMIS observes this exiting beam of electrons for some time after the wake passage, whenever the magnetic field connects to the central wake.

The top two panels of Fig. 5 show that the energy of this exiting beam correlates well with the proximity with which the magnetic field line at the spacecraft passes near the center of the wake (as estimated by a linear extrapolation of the local magnetic field direction), rather than with the position of the spacecraft itself, suggesting an origin related to the center of the wake. This beam of electrons may therefore have a source in the central wake, with the seed population then undergoing acceleration outwards by the wake electric field. However, the asymmetry of the wake clearly affects the source and/or evolution of the out-going beam, since we do not observe a similar beam on the entry side of the wake.

We can infer the wake potential structure by comparing the field-aligned components of the electron distribution function in the wake with those measured outside the wake, as demonstrated previously using Nozomi and Lunar Prospector data (Futaana et al. 2001; Halekas et al. 2005). We use a parallel electron distribution function measured just outside the entry side of the wake (at 08:36), and an anti-parallel distribution function measured just outside the exit side of the wake (at 09:43), as references to calculate shifts in parallel/antiparallel electron spectra from the entry/exit sides of the wake to the center of the wake. To accomplish this determination, we use electrons with energies from 40-200 eV (which penetrate all the way through the wake) as tracer populations to derive the potential drop into the wake. This procedure tacitly assumes that the source spectrum of solar wind electrons does not change significantly during the observation time period.

The fifth panel of Fig. 5 shows the results of this derivation, along with a model potential computed from the same self-similar model described in Sect. 4, with exactly the same parameters previously used. The model and data agree rather well, with small fluctuations in the derived potential indicating spatial and/or temporal variations not represented in the model. We find only one major difference from the model, namely an asymmetry between the potential drop derived on the entry and exit sides of the wake. The wake potential may plausibly have a real spatial asymmetry, since the refilling electron populations from the two ends of the flux tube have different properties, and the wake isolates the two ends of the flux tube from each other. The strong strahl population incident on the exit side of the wake very likely affects the plasma expansion, increasing the wake potential drop compared to that on the entry side, particularly deeper in the wake where the strahl dominates the electron density. Alternatively, this asymmetry could result from temporal changes during the wake traverse. Only two-point measurements, which ARTEMIS will provide in coming years, can settle this question conclusively, but we find strong hints that point towards the former interpretation.

By using electron distribution functions from the center of the wake (at 09:08) as references, we can similarly approximately derive the potential drop sensed by electrons traveling outwards from the central wake. This spectral shift depends on the potential drop between the local plasma and the deepest point in the wake on the magnetic field line of the spacecraft, rather than that between the solar wind and the local plasma, with our derivation assuming temporal invariance and a constant electron spectrum throughout the central 
Fig. 6 Schematic illustration of velocity filtration of the electron spectrum by the wake potential, showing distribution functions (at three points) and trajectories of core electrons, reflected core electrons, and strahl electrons
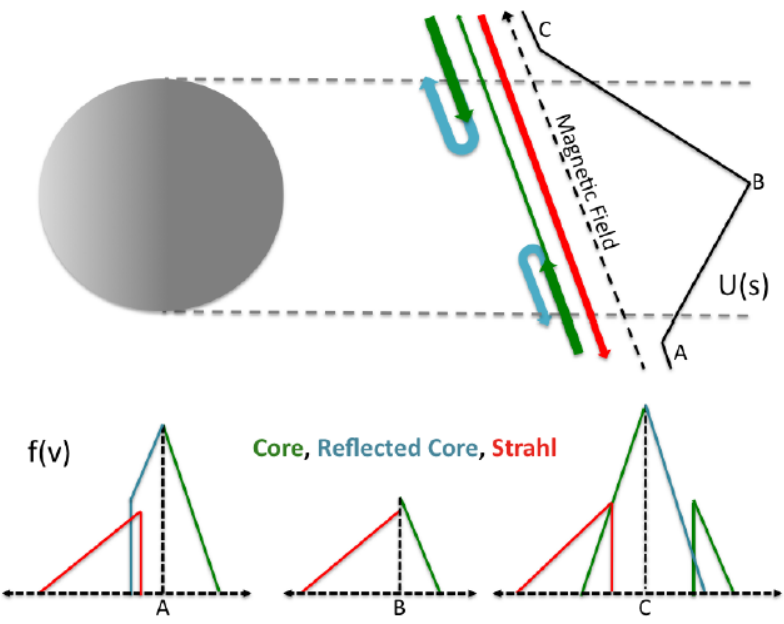

wake. Using these procedures, we can use electron distributions as a probe not only of the local wake potential, but that deeper in the wake along the spacecraft magnetic field line. As above, we compare derived potential drops with the results of our self-similar model, in the sixth panel of Fig. 5. Again, the derived potential drops agree fairly well with our model, except that the model potential drop on the entry side exceeds the actual drop sensed by electrons traveling out from that side of the wake. This implies a potential drop on the entry side of the wake smaller than expected, and smaller than that on the exit side, consistent with the results just discussed. This asymmetry could explain the lack of a clearly observable beam of electrons traveling outward from the entry side of the wake.

As a final check on our inferred potential structure, we derive synthetic spectrograms for parallel and anti-parallel electrons, using the same end-point spectra we used to derive the potentials just discussed. We manufacture these synthetic spectrograms by constructing a superposition of incident electrons, reflected electrons, and electrons accelerated out from the central wake, again using the potential drops derived above to shift the distribution functions appropriately. The resulting superposed spectra match the observed distributions very well, other than small fluctuations likely due to temporal variations. The synthetic spectrograms, based on derived potentials that rely only on measurements above $40 \mathrm{eV}$, also match the lower energy electrons, with the same basic features as the observed spectrograms. Furthermore, we find that the beam of electrons observed on the exit side of the wake matches very well with the electron spectrum measured in the central wake, shifted by the inferred potential drop from the central wake to the observation point. In other words, this exiting beam most likely consists of a residual population of core/halo electrons that penetrates through the central wake from the entry side and then undergoes acceleration outwards by the wake potential on the exit side. In some regions, this exiting beam co-exists with a reflected population of incident electrons with energies below the beam energy.

In Fig. 6, we show a schematic illustration of the tentatively identified components of the electron spectra in and around the wake, produced by the filtering and acceleration effects of the wake potential. A small portion of the electron core population traveling parallel along the magnetic field from the entry side of the wake penetrates to the central wake, where it feels the electric field set up by the plasma refilling the wake from the exit side, and undergoes acceleration outwards through the wake potential, producing the observed beam. At the same time, few core electrons penetrate from the exit side, but a strong strahl 
Fig. 7 Parallel cuts through electron distribution functions, covering the same pitch angle ranges as the spectrograms in Fig. 5, as measured at three times before (black), during (blue), and after (red) the wake crossing. Positive values on the horizontal axis represent electrons with velocity parallel to the magnetic field and negative values represent electrons with anti-parallel velocity, for ease of comparison to the schematic spectra in Fig. 6

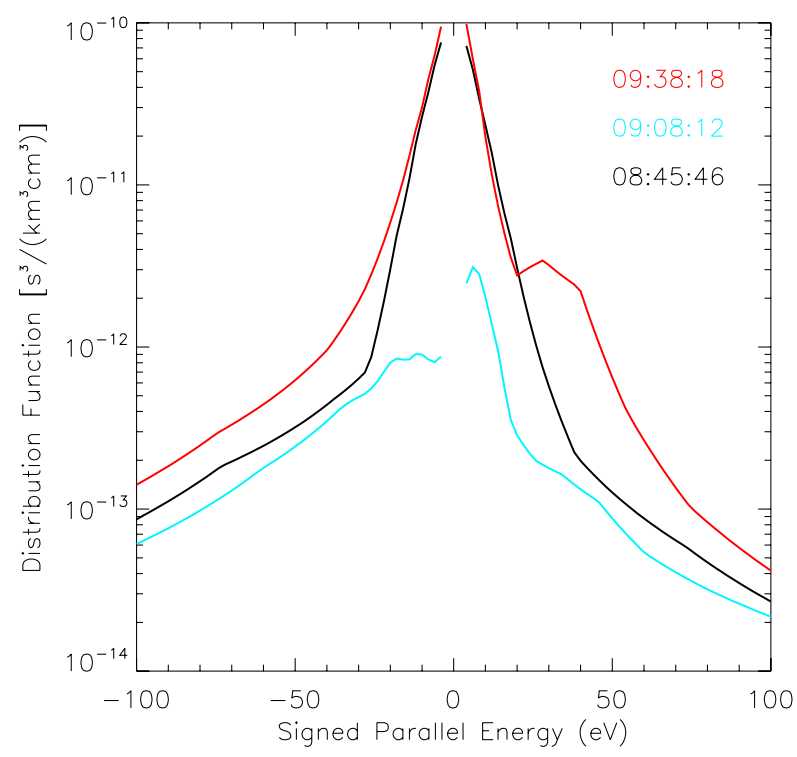

component travels all the way through the wake and out the other side. In Fig. 7, we show corresponding measured distribution function cuts, demonstrating a striking agreement to those postulated in Fig. 6. The measured spectra naturally show more complexity than the cartoon illustrations, with multiple spectral components (core and halo populations) visible in both the parallel and anti-parallel cuts. However, the key features remain, including the anti-parallel strahl enhancement, the residual parallel core population in the central wake, and the parallel beam formed by the acceleration of this residual population out of the wake. The superposition of these various electron populations plausibly explains all of the features we observe in the electron spectrograms, with no need to postulate any significant temporal variations, though we cannot rule out temporal variations without two-point measurements (which we will soon have with ARTEMIS). A key element of the explanation presented here relies on the asymmetric character of the wake, ensured naturally by the magnetic field line tilt and the different electron distributions incident on the wake from the entry and exit sides. We find it fascinating to speculate on what happens at the boundary between flux tubes connected to the wake and those not connected to the wake, given the possible presence of an asymmetric potential, in addition to differential electron/ion shadowing (Bale et al. 1997), each of which may drive instabilities.

The wake potential first filters incident solar wind electron distributions and then reaccelerates them, naturally producing electron beams exiting the wake. Previous studies have suggested the existence of such filtered distributions, based on observations of wave turbulence outside of the wake (Farrell et al. 1996; Nakagawa et al. 2003). We now have the first direct observations of the electron distributions that could drive such upstream waves. These electron beams can also drive local wave processes in the wake, as can the counterstreaming refilling ion beams. Indeed, EFI measures both broadband electrostatic noise in the central wake, and electrostatic waves near the electron plasma frequency on the exit side of the wake (see Fig. 1). Either ions or electrons may generate the broadband turbulence in the central wake, with previous predictions suggesting a role for the ions (Farrell et al. 1998). The higher frequency oscillations on the exit side of the wake, on the other hand, correlate very well with the exiting electron beam, suggesting that the beam and the oppo- 
sitely directed strahl electrons generate waves through a streaming instability. We observe these waves at a frequency slightly below the electron plasma frequency, consistent with the downshift expected when beam components comprise a significant fraction of the plasma density (Fuselier et al. 1985). Outside of the wake, core electrons may help damp wave growth, but inside the wake, where the incoming strahl and the exiting beam dominate the electron density, strong wave growth can generate the observed electrostatic oscillations. Birch and Chapman (2001a, 2001b) predicted just such electrostatic oscillations, which we have now observed with ARTEMIS. In the coming years, ARTEMIS will provide many more detailed observations of the wave-particle interactions created by the filtration effects of the wake potential.

\section{Conclusions}

On the first of many orbits, from one of two ARTEMIS probes, we have already made new and exciting observations of interpenetrating electron, proton, and alpha particle populations refilling the wake from both flanks. The wake potential driven by the refilling process filters and accelerates both ions and electrons, naturally producing unstable distributions that should generate numerous instabilities, including the electrostatic turbulence observed on this orbit. Single-point measurements already suggest the presence of significant asymmetries in the wake, resulting from tilted magnetic fields and anisotropic solar wind electron distributions. Soon, ARTEMIS's two-probe measurement capability will allow us to conclusively separate temporal and spatial effects, and determine the causal flow of many processes hinted at in these observations. ARTEMIS's comprehensive instrumentation and elliptical orbits will also allow investigations of the $3 \mathrm{~d}$ structure and dynamics of the wake and many other aspects of the lunar plasma environment, including pickup ions from the exosphere and surface, magnetic anomaly interactions, surface electric fields, and no doubt many as yet unanticipated plasma processes. ARTEMIS will also support and complement measurements from other current and planned missions such as LADEE, ILN, and LRO, providing an integral part of the lunar constellation.

Acknowledgements We wish to acknowledge the extraordinary team of scientists and engineers that made the THEMIS/ARTEMIS missions a reality. We thank R. Lepping, K. Ogilvie, and CDAWEB for providing Wind key parameter data. We also acknowledge NASA's Lunar Science Institute for supporting JSH, WMF, and GTD. FP and KHG acknowledge financial support by the German Ministerium für Wirtschaft und Technologie and the Deutsches Zentrum für Luft- und Raumfahrt under grant 50QP0402 is acknowledged.

Open Access This article is distributed under the terms of the Creative Commons Attribution Noncommercial License which permits any noncommercial use, distribution, and reproduction in any medium, provided the original author(s) and source are credited.

\section{References}

V. Angelopoulos, The THEMIS mission. Space Sci. Rev. 141, 5-34 (2008)

H.U. Auster, K.H. Glassmeier, W. Magnes, O. Avdogar, W. Baumjohann, D. Constantinescu, D. Fischer, K.H. Fornacon, E. Georgescu, P. Harvey, O. Hillenmaier, R. Kroth, M. Ludlam, Y. Narita, R. Nakamura, K. Okrafka, F. Plaschke, I. Richter, H. Schwarzl, B. Stoll, A. Valavanoglou, M. Wiedemann, The THEMIS fluxgate magnetometer. Space Sci. Rev. 141, 235-264 (2008)

S.D. Bale, C.J. Owen, J.-L. Bougeret, K. Goetz, P.J. Kellogg, R.P. Lin, R. Manning, S.J. Monson, Evidence of currents and unstable particle distributions in an extended region around the lunar wake. Geophys. Res. Lett. 24, 1427-1430 (1997) 
P.C. Birch, S.C. Chapman, Particle-in-cell simulations of the lunar wake with high phase space resolution. Geophys. Res. Lett. 28, 219 (2001a)

P.C. Birch, S.C. Chapman, Detailed structure and dynamics in particle-in-cell simulations of the lunar wake. Phys. Plasmas 8, 4551-4559 (2001b)

J.W. Bonnell, F.S. Mozer, G.T. Delory, A.J. Hull, R.E. Ergun, C.M. Cully, V. Angelopoulos, P.R. Harvey, The electric field instrument (EFI) for THEMIS. Space Sci. Rev. 141, 303-341 (2008)

D. Clack, J.C. Kasper, A.J. Lazarus, J.T. Steinberg, W.M. Farrell, Wind observations of extreme ion temperature anisotropies in the lunar wake. Geophys. Res. Lett. 31, L06812 (2004). doi:10.1029/ 2003GL018298

D.S. Colburn, R.G. Currie, J.D. Mihalov, C.P. Sonett, Diamagnetic solar-wind cavity discovered behind moon. Science 158, 1040 (1967)

J.E. Crow, P.L. Auer, J.E. Allen, The expansion of plasma into a vacuum. J. Plasma Phys. 14, 65-76 (1975)

J. Denavit, Collisionless plasma expansion into a vacuum. Phys. Fluids 22, 1384-1392 (1979)

P. Dyal, C.W. Parkin, W.D. Daily, Magnetism and the interior of the Moon. Rev. Geophys. Space Phys. 12, 568-591 (1974)

W.M. Farrell, R.J. Fitzenreiter, C.J. Owen, J.B. Byrnes, R.P. Lepping, K.W. Ogilvie, F. Neubauer, Upstream ULF waves and energetic electrons associated with the lunar wake: Detection of precursor activity. Geophys. Res. Lett. 23, 1271-1274 (1996)

W.M. Farrell, M.L. Kaiser, J.T. Steinberg, S.D. Bale, A simple simulation of a plasma void: Applications to Wind observations of the lunar wake. J. Geophys. Res. 103, 23653-23660 (1998)

J.W. Freeman Jr., M.A. Fenner, H.K. Hills, Electric potential of the Moon in the solar wind. J. Geophys. Res. 78, 4560-4567 (1973)

S.A. Fuselier, D.A. Gurnett, R.J. Fitzenreiter, The downshift of electron plasma oscillations in the electron foreshock region. J. Geophys. Res. 90, 3935-3946 (1985)

Y. Futaana, S. Machida, Y. Saito, A. Matsuoka, H. Hayakawa, Counterstreaming electrons in the near vicinity of the Moon observed by plasma instruments on board NOZOMI. J. Geophys. Res. 106, 18729-28740 (2001)

Y. Futaana, S. Barabash, M. Weiser, M. Holmstrom, A. Bhardwaj, M.B. Dhanya, R. Sridharan, P. Wurz, A. Schaufelberger, K. Asamura, Protons in the near lunar wake observed by the Sub-keV Atom Reflection Analyzer on board Chandrayaan-1. J. Geophys. Res. 115, A10248 (2010). doi:10.1029/2010JA015264

J.S. Halekas, S.D. Bale, D.L. Mitchell, R.P. Lin, Magnetic fields and electrons in the lunar plasma wake. J. Geophys. Res. 110, A07222 (2005). doi:10.1029/2004JA010991

J.S. Halekas, G.T. Delory, D.A. Brain, R.P. Lin, D.L. Mitchell, Density cavity observed over a strong lunar crustal magnetic anomaly in the solar wind: A mini-magnetosphere? Planet. Space Sci. 56/7, 941-946 (2008a). doi:10.1016/j.pss.2008.01.008

J.S. Halekas, G.T. Delory, R.P. Lin, T.J. Stubbs, W.M. Farrell, Lunar Prospector observations of the electrostatic potential of the lunar surface and its response to incident currents. J. Geophys. Res. 113, A09102 (2008b). doi:10.1029/2008JA013194

M. Holmström, M. Weiser, S. Barabash, Y. Futaana, A. Bhardwaj, Dynamics of solar wind protons reflected by the Moon. J. Geophys. Res. (2010). doi:10.1029/2009JA014843

E. Kallio, Formation of the lunar wake in quasi-neutral hybrid model. Geophys. Res. Lett. 32, L06107 (2005). doi:10.1029/2004GL021989

S. Kimura, T. Nakagawa, Electromagnetic full particle simulation of the electric field structure around the moon and the lunar wake. Earth Planets Space 60, 591-599 (2008)

R.P. Lin, D.L. Mitchell, D.W. Curtis, K.A. Anderson, C.W. Carlson, J. McFadden, M.H. Acuña, L.L. Hood, A. Binder, Lunar surface magnetic fields and their interaction with the solar wind: Results from Lunar Prospector. Science 281, 1480-1484 (1998)

J.P. McFadden, C.W. Carlson, D. Larson, M. Ludlam, R. Abiad, B. Elliott, P. Turin, M. Marckwordt, V. Angelopoulos, The THEMIS ESA plasma instrument and in-flight calibration. Space Sci. Rev. 141, 277-302 (2008)

T. Nakagawa, Y. Takahashi, M. Iizima, GEOTAIL observation of upstream ULF waves associated with the lunar wake. Earth Planets Space 55, 569-580 (2003)

N.F. Ness, Interaction of the solar wind with the Moon, in Solar Terrestrial Physics/1970, Part II, ed. by E.R. Dyer (Reidel, Dordrecht, 1972), pp. 159-205

N.F. Ness, K.W. Behannon, C.S. Searce, S.C. Cantarano, Early results from the magnetic field instrument on Lunar Explorer 35. J. Geophys. Res. 72, 5769-5778 (1967)

M.N. Nishino, K. Maezawa, M. Fujimoto, Y. Saito, S. Yokota, K. Asamura, T. Tanaka, H. Tsunakawa, M. Matsushima, F. Takahashi, T. Terasawa, H. Shibuya, H. Shimizu, Pairwise energy gain-loss feature of solar wind protons in the near-Moon wake. Geophys. Res. Lett. 36, L12108 (2009a). doi:10.1029/2009GL039049 
M.N. Nishino, M. Fujimoto, K. Maezawa, Y. Saito, S. Yokota, K. Asamura, T. Tanaka, H. Tsunakawa, M. Matsushima, F. Takahashi, T. Terasawa, H. Shibuya, H. Shimizu, Solar-wind proton access deep into the near-Moon wake. Geophys. Res. Lett. 36, L16103 (2009b). doi:10.1029/2009GL039444

K.W. Ogilvie, J.T. Steinberg, R.J. Fitzenreiter, C.J. Owen, A.J. Lazarus, W.M. Farrell, R.B. Torbert, Observations of the lunar plasma wake from the WIND spacecraft on December 27, 1994. Geophys. Res. Lett. 10, 1255-1258 (1996)

C.J. Owen, R.P. Lepping, K.W. Ogilvie, J.A. Slavin, W.M. Farrell, J.B. Byrnes, The lunar wake at $6.8 R_{L}$ : WIND magnetic field observations. Geophys. Res. Lett. 10, 1263-1266 (1996)

A. Roux, O. le Contel, C. Coillot, A. Bouabdellah, B. de la Porte, D. Alison, S. Ruocco, M.C. Vassal, The search coil magnetometer for THEMIS. Space Sci. Rev. 141, 265-275 (2008)

C.T. Russell, B.R. Lichtenstein, On the source of lunar limb compression. J. Geophys. Res. 80, 4700 (1975)

Y. Saito, S. Yokota, T. Tanaka, K. Asamura, M.N. Nishino, M. Fujimoto, H. Tsunakawa, H. Shibuya, M. Matsushima, H. Shimizu, F. Takahashi, T. Mukai, T. Terasawa, Solar wind proton reflection at the lunar surface: Low energy ion measurements by MAP-PACE onboard SELENE (KAGUYA). Geophys. Res. Lett. 35, L24205 (2008). doi:10.1029/2008GL036077

Y. Saito, S. Yokota, K. Asamura, T. Tanaka, M.N. Nishino, T. Yamamoto, Y. Terakawa, M. Fujimoto, H. Hasegawa, H. Hayakawa, M. Hirahara, M. Hoshino, S. Machida, T. Mukai, T. Nagai, T. Nagatsuma, T. Nakagawa, M. Nakamura, K. Oyama, E. Sagawa, S. Sasaki, K. Seki, I. Shinohara, T. Terasawa, H. Tsunakawa, H. Shibuya, M. Matsushima, H. Shimizu, F. Takahashi, In-flight performance and initial results of Plasma energy Angle and Composition Experiment (PACE) on SELENE (Kaguya). Space Sci. Rev. 154(1-4), 265-303 (2010). doi:10.1007/s11214-010-9647-x

U. Samir, K.H. Wright Jr., N.H. Stone, The expansion of a plasma into a vacuum: Basic phenomena and processes and applications to space plasma physics. Rev. Geophys. 21, 1631-1646 (1983)

G. Schubert, B.R. Lichtenstein, Observations of Moon-plasma interactions by orbital and surface experiments. Rev. Geophys. 12, 592-626 (1974)

N. Singh, R.W. Schunk, Numerical calculations relevant to the initial expansion of the polar wind. J. Geophys. Res. 87, 9154-9170 (1982)

P. Trávnicek, P. Hellinger, Structure of the lunar wake: Two-dimensional global hybrid simulations. Geophys. Res. Lett. 32, L06102 (2005). doi:10.1029/2004GL022243

X.-D. Wang, W. Bian, J.-S. Wang, J.-J. Liu, Y.-L. Zou, H.-B. Zhang, C. Lü, J.-Z. Liu, W. Zuo, Y. Su, W.B. Wen, M. Wang, Z.-Y. Ouyang, C.-L. Li, Acceleration of scattered solar wind protons at the polar terminator of the Moon: Results from Chang'E-1/SWIDs. Geophys. Res. Lett. 37, L07203 (2010). doi:10.1029/2010GL042891

M. Wieser, S. Barabash, Y. Futaana, M. Holmstrom, A. Bhardwaj, R. Sridharan, M.B. Dhanya, P. Wurz, A. Schaufelberger, K. Asamura, Extremely high reflection of solar wind protons as neutral hydrogen atoms from regolith in space. Planet. Space Sci. (2009). doi:10.1016/j.pss.2009.09.012

M. Wieser, S. Barabash, Y. Futaana, M. Holmstrom, A. Bhardwaj, R. Sridharan, M.B. Dhanya, P. Wurz, A. Schaufelberger, K. Asamura, First observation of a mini-magnetosphere above a lunar magnetic anomaly using energetic neutral atoms. Geophys. Res. Lett. 37, L015103 (2010). doi:10.1029/ 2009GL041721 